\title{
Mineral trióxido agregado en pulpotomías de dientes primarios. Revisión de la literatura
}

\section{Mineral trioxide aggregate in primary teeth pulpotomy. Literature review}

\author{
Simancas Pallares M*, Luna Ricardo L**, Díaz Caballero A***
}

\section{RESUMEN}

Introducción: la odontología basada en la evidencia supone una evaluación, crítica, de la evidencia disponible para mejorar la toma de decisiones en relación con el cuidado individual de los pacientes y/o comunidades. Objetivo: analizar la literatura científica disponible sobre los resultados clínicos y radiográficos de cuatro materiales empleados en pulpotomías en la dentición temporal: formocresol, sulfato férrico, hidróxido de calcio, mineral trióxido agregado. Materiales y métodos: se identificaron las publicaciones más relevantes a través de una búsqueda en bases de datos electrónicas como MEDLINE (Ovid) y "The Cochrane Library". Para ser incluidos en la revisión, los estudios debieron definir el material utilizado, en pacientes niños con exposición pulpar por caries o trauma dentoalveolar. Resultados: de los 21 artículos obtenidos en la fase inicial de la revisión, sólo 19 estaban disponibles en texto completo y de éstos sólo cumplieron los requisitos de inclusión 6 artículos, los cuales fueron confrontados, analizados y discutidos posteriormente. Conclusiones: la evidencia disponible demuestra que no existen diferencias clínicas en cuanto a la utilización de un material u otro, sin embargo se deben tener en cuenta otras variables como factores económicos y edad del paciente para la correcta terapéutica.

Palabras clave: Pulpotomía, pulpa dental, ensayo clínico controlado aleatorio.

\section{SUMMARY}

Introduction: evidence-based dentistry is a critical evaluation, raise awareness of the available evidence to improve decision making about the care of individual patients and / or communities. Objective: to analyze the available scientific literature on clinical and radiographic outcomes of two materials used in pulpotomy in primary teeth: formocresol and mineral trioxide aggregate. Materials and methods: it was identified relevant publications through a search of electronic databases such as MEDLINE (Ovid) and The Cochrane Library. To be included in the review, studies had to define the material used in child patients with pulp exposure by caries or tooth-alveolar trauma. Results: of the 21 items obtained in the initial phase of the review, only 19 were available in full text and of these only met the requirements for inclusion 6 items, which were confronted, analyzed and discussed later. Conclusions: the available evidence shows that there are no clinical differences regarding the use of a material or another, but must take into account other variables such as economic factors and patient age for proper treatment.

Key words: Pulpotomy, dental pulp, randomized controlled trial.

Fecha de recepción: 16 de octubre de 2009.

Aceptado para publicación: 20 de octubre de 2009.

* Odontólogo. Facultad de Odontología. Universidad de Cartagena.

** Odontóloga. Especialista en Odontología Integral del Niño. Docente Titular Facultad de Odontología. Universidad de Cartagena.

*** Odontólogo. Candidato a Doctor en Ciencias Biomédicas.

Simancas Pallares M, Luna Ricardo L, Díaz Caballero A. Mineral trióxido agregado en pulpotomías de dientes primarios. Revisión de la literatura. Av. Odontoestomatol 2011; 27 (2): 91-98. 


\section{INTRODUCCIÓN}

El progreso en el entendimiento de los cambios moleculares y celulares durante el desarrollo dental y cómo son imitados durante la reparación tisular, ofrecen la oportunidad de evaluar la validez biológica de varias estrategias de tratamiento pulpar vital. Bajo esta perspectiva se ofrecen opciones diferentes de terapia pulpar como el tratamiento pulpar indirecto (T.P.I), directo (T.P.D), pulpotomía, pulpectomía basados en una buena historia clínica para obtener un buen diagnóstico (1).

Si un diente con caries permanece sin tratar o tratado inadecuadamente, ocurrirá invasión bacteriana a la pulpa coronal dando como resultado una respuesta inflamatoria a ese nivel. En este estadio la inflamación se encuentra confinada a este espacio; pero si el tejido afectado se remueve y la entrada de los canales radiculares se cubre con un agente apropiado, el remanente tisular tiene la capacidad de recuperarse. Esta capacidad se utiliza cuando un molar con exposición pulpar se trata con terapia pulpar vital. De esta manera el objetivo principal del tratamiento pulpar es mantener la integridad y salud de los tejidos orales. Sin embargo un diente puede continuar funcional sin una pulpa coronal vital; es deseable intentar mantener la vitalidad de la pulpa, así el objetivo de la pulpotomía vital es tratar la injuria reversible (2).

La pulpotomía es uno de los tratamientos frecuentemente usados para conservar molares primarios con compromiso carioso, libres de sintomatología o con pulpitis reversible que de otra manera serían extraídos. Su objetivo es preservar la pulpa radicular, evitar el dolor y la inflamación y mantener el diente (3).

El formocresol (FC) se utilizó por primera vez en 1930 por Sweet quién reportó una tasa de éxito de $97 \%$. Produce un área de necrosis en la pulpa adyacente a la lesión. A menudo el tejido se altera por el formaldehído y aparece "fijado" in situ y por tanto no se somete inmediatamente a necrosis licuefactiva en el canal radicular. Es el agente de recubrimiento pulpar para molares primarios más utilizado, sin embargo el formaldehído el mayor componente del formocresol, muestra cierto riesgo de distribución sistémica después de su uso (4). Recientemente se expresan preocupaciones con respecto a la pulpotomía con formocresol debido a que se observa: respuesta pulpar con inflamación y necrosis, citotoxicidad, disturbios sistémicos, potencial mutagenicidad y carcinogenicidad así como también respuesta inmune alterada.

No obstante con el devenir de la tecnología se investigan diferentes compuestos con el fin de hallar el que provea mayor eficacia clínica sin efectos colaterales, entre estos figuran ampliamente en la literatura científica: electrocirugía, láser Er: YAG, hidróxido de calcio, glutaraldehído, sulfato férrico, solución enriquecida de colágeno, proteínas morfogenéticas óseas o mineral trióxido agregado (M.T.A); sin embargo esta se revisión se limita exclusivamente a comparar el formocresol $(\mathrm{FC})$ con el mineral trióxido agregado (M.T.A) (3).

El objetivo de ésta revisión sistemática de la literatura es comparar la evidencia disponible sobre los efectos clínicos y radiográficos del formocresol comparado con el mineral trióxido agregado en molares primarios tratados con pulpotomía.

\section{MATERIALES Y MÉTODOS}

Se realizó una búsqueda computarizada de literatura utilizando MEDLINE (Ovid: 1960-2008) (Tabla 1), y The Cochrane Library. Se seleccionaron ensayos controlados randomizados en donde se comparara técnica de pulpotomía en dientes primarios con formocresol y/o mineral trióxido agregado. Se evaluaron los títulos y resúmenes de estudios de acuerdo a su relevancia después de obtener los artículos en texto completo. La búsqueda terminó en Enero de 2009 .

Los criterios de inclusión fueron: estudios que evaluaran tratamiento pulpar de molares primarios con exposición pulpar vital por caries o trauma que incluyeran materiales como formocresol y/o mineral trióxido agregado, tiempo de seguimiento de 6 meses mínimo, dientes sin sintomatología que indicara daño irreversible, dientes restaurables, evaluación de resultados por métodos clínicos y radiográficos. Criterios de exclusión: no aleatorización de sujetos, estudios realizados in vitro o en animales, no realización 


\section{TABLA 1.- ESTRATEGIA DE BÚSQUEDA EN} MEDLINE (OVID). FC: FORMOCRESOL

\begin{tabular}{|r|l|r|}
\hline$N^{\circ}$ & \multicolumn{1}{|c|}{ Palabras clave } & Resultados \\
\hline 1 & Pulpotomy & 296 \\
2 & Pulp therapy & 179 \\
3 & Primary molar & 290 \\
4 & Mineral trioxide aggregate & 546 \\
5 & 1 and 3 & 65 \\
6 & 1 or 3 & 521 \\
7 & 4 and 1 and 3 & 41 \\
8 & 4 or 1 or 3 & 567 \\
9 & 4 and 1 and 3 and 5 & 20 \\
10 & 4 or 1 or 3 or 5 & 595 \\
11 & 6 and 4 and 1 and 3 and 5 & 15 \\
12 & 6 or 4 or 1 or 3 or 5 & 1.021 \\
13 & 7 and 1 and 3 and 5 & 8 \\
14 & 7 or 1 or 3 or 5 & 7.222 \\
15 & FC & 7.668 \\
16 & 13 and 15 & 21 \\
17 & 15 and 14 and 16 & \\
\hline
\end{tabular}

de comparaciones entre los grupos de tratamiento en el estudio, el artículo no pudo ser localizado.

Inicialmente se obtuvo 21 artículos para posible inclusión, aplicando los criterios se seleccionaron 6 artículos (ensayos clínicos controlados aleatorizados o ensayos controlados randomizados) para posterior análisis. Ver Tabla 1.

Posterior a esto los artículos se indexaron en una base de datos digital, se procedió al análisis de cada uno de estos tomando como datos referenciales los autores, el título, revista, año, volumen, número, mes, páginas. Se detalló la metodología empleada y también se hizo énfasis en los resultados (número de dientes, tasa de éxito, tasa de fracaso, frecuencias). Se confrontaron y analizaron sus resultados para ser posteriormente discutidos.

\section{RESULTADOS}

El formocresol se introdujo en 1904 (5) gracias a Buckley y desde entonces se considera el "patrón de oro" con el cual los demás medicamentos deben compararse en experimentos de pulpotomías de molares primarios. Se convirtió en el medicamento más usado, debido a sus propiedades bacteriostáticas y fijativas, con una tasa de éxito que varía entre el 55 al 98\%. Sin embargo otros autores sugieren que la eficacia clínica y radiográfica del formocresol varia entre el 70 al $97 \%$ (6). Su composición química de formaldehído (mutagénico y carcinógeno) y cresol (agente cáustico), suscita preocupación, aún cuando su distribución sistémica no posee evidencia de causar enfermedades (7).

La respuesta histológica de la pulpa después de la colocación del formocresol parece ser desfavorable. Algunos investigadores afirman que después de la aplicación del formocresol, ocurre fijación en el tercio coronal de la pulpa radicular e inflamación crónica en el tercio medio, quedando inalterado o vital el tercio apical. Otros reportan que el tejido pulpar remanente está parcial o totalmente necrótico. Comúnmente, la pulpa queda mitad vital, mitad necrótica e inflamada crónicamente. Aunque muchos estudios publicados durante la década de los 90's cuestionaron la seguridad y eficacia del formocresol muchas autoridades acuerdan que es potencialmente mutagénico, es el material de elección para pulpotomías en la dentición temporal.

Sin embargo la Agencia Internacional para la Investigación en Cáncer (IARC: International Agency for Research on Cancer), evaluando los documentos disponibles, concluyeron que existe suficiente evidencia en experimentos animales y en humanos para clasificar el formaldehído como carcinógeno. La declaración de la IARC recomendó la sustitución de los materiales que contengan formaldehído por alternativas más seguras (8).

A pesar de los esfuerzos científicos que se realizan en la búsqueda de un material de reemplazo adecuado para el FC, las investigaciones sobre un material novedoso denominado Mineral Trióxido agregado (M.T.A) desarrollado por Torabinejad y colaboradores en la Universidad de Loma Linda (Loma Linda University, CA, USA) aproximadamente en 1995 (9), sugieren resultados excelentes cuando se utiliza en pulpotomías en dientes primarios. 
Solamente hasta 1998, la Administración de Drogas y Alimentos de los Estados Unidos (United States Food and Drug Administration) aprobó el M.T.A como un material de terapéutica endodóntica en humanos (10). El M.T.A es un polvo compuesto de silicato tricálcico, oxido de bismuto, silicato de dicalcio, aluminato tetracálcico, aluminoferrato tetracálcico y sulfato de calcio dihidratado.

En estudios histológicos Torabinejad et al (11), utilizaron MTA para reparación de perforaciones endodónticas; el material demostró biocompatibilidad y muy poca inflamación, aún cuando el material extruyó del sitio de la perforación.

Recientemente, el MTA se introdujo como un potencial agente alternativo al formocresol con las características de liberación de citoquinas de las células óseas, inducción de formación de tejido duro, efecto dentinogénico sobre la pulpa, propiedades antimicrobianas y el mantenimiento de la integridad pulpar después del recubrimiento pulpar o la pulpotomía sin efectos citotóxicos (12).

Agamy et al en 2004 (13), en un ensayo controlado randomizado seleccionaron 72 molares en 24 niños para el estudio clínico y radiográfico. Los dientes seleccionados fueron aleatoriamente asignados y divididos en tres grupos de acuerdo al material de relleno pulpar usado (MTA gris, MTA blanco o formocresol). Un adicional de 15 dientes se preparó para extracciones seriadas y realizar un estudio histológico. Las pulpotomías se realizaron por el mismo operador y la valoración de los resultados efectuada por dos odontólogos pediatras "cegados".

Obtuvieron como resultados que todos los 60 dientes se valoraron como éxitos clínicos y radiográficos al primer mes de evaluación postoperatoria. A los 3 meses un diente del grupo del MTA blanco fue valorado como falla clínica y radiográfica, debido a un absceso, y el diente fue extraído. Los 59 dientes restantes fueron valorados como éxito clínico y radiográfico a los 3 meses postoperatorios. A los 6 meses de evaluación, 1 diente del grupo del MTA gris fue perdido debido a exfoliación y los 58 dientes restantes fueron valorados como exitosos clínica y radiográficamente.
A los 12 meses de evaluación, los 19 dientes del grupo de MTA gris fueron valorados todos como éxito clínico y radiográfico. En el grupo de MTA blanco, 3 dientes fueron valorados como fallas clínicas y radiográficas. Los 16 dientes restantes en el grupo de MTA gris fueron valorados como éxito clínico y radiográfico. Dos dientes en el grupo de formocresol fueron valorados como fallas clínicas y radiográficas. Al aplicar pruebas estadísticas no se obtuvo diferencias significativas entre un material y otro.

Farsi et al en 2005 (14) en un ensayo controlado randomizado comparan los resultados clínicos y radiográficos de dientes molares primarios con pulpotomías con formocresol o MTA, seleccionaron 100 niños aleatoriamente en el estudio. Un total de 120 dientes fueron incluidos y la pulpa coronal fue amputada usando técnica convencional de pulpotomía. Todos los grupos fueron expuestos al mismo protocolo excepto en la aplicación del material. Los dientes fueron divididos en 2 grupos iguales, en un grupo el MTA se usó como material de relleno y el FC se usó en el otro grupo.

Obtienen como resultados que de los 120 dientes, sólo 74 fueron valorados clínica y radiográficamente a través del período de seguimiento (24 meses). A lo largo de los primeros 12 meses, no se registraron patologías clínicas o radiográficas en ningún grupo. La patología radiográfica fue evidente en 4 casos de los $38(10,51 \%)$, tratados con FC, mientras que no fueron evidentes síntomas clínicos de falla en este estadio. De los casos reportados como falla, 3 dientes mostraron reabsorción interna y un diente mostró compromiso de la furcación.

Transcurridos 12 meses los resultados demuestran que ninguno de los dientes tratados con MTA presentó alguna patología radiográfica ni signo clínico de falla. La diferencia, sin embargo, no fue estadísticamente significativa $(p=0,64)$.

Pasados 24 meses el grupo de FC mostró 5 casos de patología pulpar $(13,2 \%)$, con un solo caso reportado de dolor. Todas las fallas reportadas mostraron reabsorción interna, dos de ellos mostraron compromiso de la furca. Por otro lado, todos los 38 dientes tratados con MTA fueron considerados como exitosos radiográfica y clínicamente. La diferencia 
estadística entre los 2 grupos después de 24 meses de evaluación fue evidente en cuanto a la patología radiográfica $(\mathrm{p}=0,031)$.

Holan et al en 2005 (15) en un estudio comparativo de pulpotomías con formocresol o MTA seleccionaron un total de 64 molares primarios que recibieron pulpotomías en 35 niños asignados aleatoriamente a través del tiro de una moneda a cualquiera de los dos grupos. En caso de que un niño tuviese dos molares necesitando pulpotomía, el segundo era asignado al grupo alternativo. Si un niño tuviese 3 molares indicados para pulpotomía el material de recubrimiento era designado nuevamente por el tiro de una moneda. Los niños regresaron para reexaminación cada 6 meses durante un promedio de 38 meses.

Obtienen como resultado que de los 64 molares pulpotomizados, 62 dientes en 33 niños estuvieron disponibles para análisis de tasas de éxito/fracaso. Dos molares en 2 pacientes, ambos del grupo de FC, fueron excluidos del estudio debido a que los pacientes nunca regresaron para examen de seguimiento. El material de recubrimiento que se usó fue MTA en 33 molares y FC en 29 molares. La falla en el tratamiento se detectó en 6 dientes después de un período promedio de 16 meses. 29 dientes con tratamiento exitoso fueron seguidos hasta su normal exfoliación con un periodo promedio de seguimiento de 33 meses. Los otros 27 dientes tratados exitosamente tuvieron un periodo promedio de seguimiento de 49 meses sin ninguna diferencia entre el grupo control/experimental.

La tasa de éxito de las pulpotomías de todos los dientes en este estudio fue $90 \%$; el MTA fue exitoso en $97 \%$ (32/33) y el FC tuvo un 83\% (24/29) sin diferencia estadística entre los dos materiales. El seguimiento reveló 6 fallas (10\%): 5 de ellas en el grupo de FC y 1 en MTA.

El hallazgo radiográfico más común entre todos los dientes fue la obliteración de los canales radiculares $55 \%$ (34/62). Se encontró en el 58\% de los dientes tratados con MTA (19/33) y en el 52\% de los dientes tratados con FC (15/29). Le sigue la reabsorción interna observada en 8 dientes ( 6 de FC y 2 con MTA). Por último el hallazgo menos frecuente fue el puente dentinario detectado en un diente tratado con MTA.
Naik y Hedge en 2005 (16) a través de un ensayo controlado randomizado incluyeron 50 molares primarios asintomáticos con lesiones cariosas profundas asignados aleatoriamente a tratamiento con formocresol o MTA (25 dientes cada grupo). Todos los dientes fueron restaurados con coronas de acero inoxidable y se realizó seguimiento clínico y radiográfico por seis meses (controles 1-3-6).

Obtienen como resultados que de los cincuenta (50) dientes seleccionados, 3 no estuvieron disponibles para posterior seguimiento después del primer mes. El seguimiento después del primer, tercer y sexto mes no reveló ningún hallazgo patológico clínico o radiográfico en el resto de los 47 dientes, por tanto no se realizó análisis estadístico debido al éxito de los tratamientos. El único hallazgo significativo fue el cambio de color del $60 \%$ de los dientes en donde el MTA se aplicó como medicamento después de 24 horas, pero el cual fue enmascarado posteriormente por la restauración con corona de acero inoxidable.

Peng et al en 2006 (17) en una revisión de literatura aplicando meta-análisis comparando el FC y el MTA en seis artículos valorados a través de la escala de Jadad obtienen como resultado que hubo diferencia estadísticamente significativa entre las tasas de éxito del FC y MTA en molares tratados con pulpotomía. Las valoraciones clínicas y radiográficas sugieren que el MTA fue superior al FC resultando en una menor tasa de falla. Concluyen que el MTA induce menor respuesta indeseable y puede ser un buen sustituto del FC.

Aeinehchi et al en 2007 (18) en un estudio controlado randomizado comparando los efectos del MTA y los del FC en molares primarios con pulpotomía seleccionaron 126 niños entre 5 y 9 años, quienes requerían pulpotomía. Los pacientes fueron asignados por un número aleatorio produciendo sistemáticamente el grupo control o experimental. En total 75 pacientes fueron ubicados al grupo de formocresol comparado con 51 en el grupo de MTA.

De los 126 pacientes, 26 no atendió a los seguimientos (18 FC y 8 MTA), resultando en 100 casos para análisis. A los 3 meses de seguimiento no se observaron signos de falla en los dos grupos. Una evaluación radiográfica de los dientes mostró reab- 
sorción radicular en 1 de los 57 dientes en el grupo de FC. El mismo fenómeno no fue detectado en el grupo de MTA. Después de 6 meses de seguimiento no hubo falla clínica, sin embargo en 6 de los 57 casos tratados en el FC, se detectó reabsorción radicular. En el grupo de MTA, no se observaron tales características. Las diferencias entre los grupos fueron estadísticamente significativas.

Noorollahian et al en 2008 (19) en un estudio clínico controlado aleatorizado comparando los resultados del FC con los del MTA en dientes primarios tratados con pulpotomía seleccionaron 46 niños con edades comprendidas entre los 5 y 7 años. 60 segundos molares primarios inferiores fueron tratados por una técnica (FC - MTA) de pulpotomía. Los dientes fueron aleatoriamente asignados al grupo control o experimental (30 dientes c/u) por una tabla aleatorizada de números. Los datos fueron analizados durante 24 meses para valorar la tasa de éxito de los tratamientos en los varios períodos de seguimiento $(6,12$, y 24 meses).

Obtienen como resultados a los 6 meses de seguimiento: 43 niños con 56 dientes llegaron para evaluación postoperatoria. Todos los dientes tratados en los dos grupos $(\mathrm{FC}=27-\mathrm{MTA}=29)$ fueron clínica y radiográficamente exitosos.

En los doce meses de seguimiento: 41 niños con 53 dientes llegaron para evaluación clínica y radiográfica. Los dientes tratados en el grupo de formocresol (24) fueron clínica y radiográficamente exitosos. Los dientes tratados en el grupo de MTA (29) fueron exitosos clínicamente pero el examen radiográfico reveló una falla, compromiso de furca.

En los veinticuatro meses de seguimiento: 27 niños con 36 dientes llegaron para evaluación postoperatoria. Los dientes tratados en el grupo de formocresol (18) fueron clínica y radiográficamente exitosos. Los dientes tratados en el grupo de MTA (18) fueron clínicamente exitosos. El seguimiento radiográfico reveló una falla, compromiso de furca, en otro de los molares tratados con MTA. La obliteración de los canales pulpares se detectó en uno de los dientes tratados con MTA y en 4 de los dientes tratados con formocresol. Ver tabla 2.

\section{CONCLUSIONES}

La literatura disponible actualmente supone una gran herramienta para la racionalización de la toma de decisiones clínicas correctas. Es por esto que los esfuerzos científicos por mejorar cada día la clínica no paran y es de esa manera que se introducen nuevos conceptos y en el ámbito de la terapia pulpar en Odontopediatría, nuevos materiales más compatibles con el organismo, con menor tasa de efectos sistémicos colaterales y mayor posibilidad de reparación tisular semejante a la estructura dentaria.

Basados en la evidencia científica que se encontró hasta el momento, en molares primarios humanos

TABLA 2.- EVIDENCIA QUE COMPARA RESULTADOS DE PULPOTOMÍAS CON FORMOCRESOL (FC) VS PULPOTOMÍAS CON MINERAL TRIÓXIDO AGREGADO (MTA)

\begin{tabular}{|c|c|c|c|c|c|c|c|c|c|}
\hline \multirow[b]{2}{*}{ Artículo } & \multirow[b]{2}{*}{ Edad } & \multicolumn{2}{|c|}{ MOLARES TTD. } & \multicolumn{2}{|c|}{ REsultado Cl. } & \multicolumn{2}{|c|}{ RESULTADO RX. } & \multirow{2}{*}{$\frac{\text { TIEMPO }}{\text { Meses }}$} & \multirow[t]{2}{*}{ AlEAT. } \\
\hline & & FC & MTA & FC & MTA & FC & MTA & & \\
\hline Agamy et al, 2004 . & $6,1 \mathrm{~A}$ & 20 & 20 & 18 & 19 & 18 & 19 & 12 & + \\
\hline Holan et al, 2005. & $6,2 \mathrm{~A}$ & 29 & 33 & 24 & 32 & 24 & 32 & $\leq 74$ & + \\
\hline Farsi et al, 2005. & $6,0 \mathrm{~A}$ & 36 & 38 & 35 & 38 & 31 & 38 & 24 & + \\
\hline Naik y Hedge, 2005. & N.E. & 23 & 24 & 24 & 24 & 23 & 24 & 6 & + \\
\hline Aienechchi et al 2005. & $6,4 \mathrm{~A}$ & 57 & 43 & 57 & 43 & 47 & 43 & 6 & + \\
\hline Noorollahian, 2008. & $6,0 \mathrm{~A}$ & 27 & 29 & 18 & 18 & 18 & 17 & 24 & + \\
\hline
\end{tabular}

Molares Ttd: número de dientes molares tratados con cada técnica. Resultado Cl: Frecuencia de éxito Clínico. Resultado Rx: Frecuencia de éxito radiográfico. Tiempo (Meses): Cantidad de meses de seguimiento. Aleat: Fue el estudio aleatorizado? (+): Si (-):No. 
con pulpitis reversible, con exposición pulpar por caries o trauma dentoalveolar es posible realizar una pulpotomía con formocresol o mineral trióxido agregado, con tasas de éxito clínico similar. Sin embargo dada la frecuencia de aparición de signos radiográficos como lesiones de furcación y su cuestionable toxicidad, con respecto a la utilización del formocresol los autores sugieren la aplicación mineral trióxido agregado teniendo en cuenta otras variables como la edad del paciente, presencia o no del diente permanente sucesor, factor socioeconómico que conllevan a tratamientos exitosos.

\section{AGRADECIMIENTOS}

El autor agradece a: Dr. Eliecer Eidelman. Profesor de la Escuela de Medicina Dental de la Universidad Hebrea de Jerusalem, a la Dra. Kaaren Vargas, profesora asociada, Universidad de Iowa, USA, por su valiosa colaboración en la lectura del documento, sugerencias y todo el apoyo brindado.

\section{BIBLIOGRAFÍA}

1. Fuks AB. Current concepts in vital primary pulp therapy. Eur J Paediatr Dent. 2002 Sep;3(3): 115-20.

2. Tziafas D, Smith AJ, Lesot H. Designing new treatment strategies in vital pulp therapy. J Dent 2000 Feb;28(2):77-92.

3. American Academy of Pediatric Dentistry. Guideline on pulp therapy for primary and young permanent teeth. Pediatr Dent. 2004;26(7 Suppl): $115-9$.

4. Sweet CA. Procedure for treatment of exposed pulpless deciduous teeth. J Am Dent Assoc. 1930;17:1150-4.

5. Buckley JP. The chemistry of pulp decomposition, with a rational treatment for this condition and its sequelae. Am Dent J. 1904;3:764-771.

6. Sonmez D, Sari S, Cetinbas T. A Comparison of four pulpotomy techniques in primary molars: a long-term follow-up. J Endod. 2008 Aug;34(8): 950-5.

7. Burnett S, Walker J. Comparison of ferric sulfate, formocresol, and a combination of ferric sulfate/ formocresol in primary tooth vital pulpotomies: a retrospective radiographic survey. ASDC J Dent Child. 2002 Jan-Apr;69(1):44-8, 12.

8. International Agency for Research on Cancer, World Health Organization. Disponible en: http:/ /www.iarc.fr/en/media-centre/pr/2004/pr153.html; Julio 10 de 2009.

9. Torabinejad M, Hong CU, McDonald F, Pitt Ford TR. Physical and chemical properties of a new root-end filling material. J Endod. 1995 Jul;21(7): 349-53.

10. Schwartz RS, Mauger M, Clement DJ, Walker WA 3rd. Mineral trioxide aggregate: a new material for endodontics. J Am Dent Assoc. 1999 Jul; 130 (7):967-75.

11. Torabinejad M, Pitt Ford TR, McKendry DJ, Abedi HR, Miller DA, Kariyawasam SP. Histologic assessment of mineral trioxide aggregate as a root-end filling in monkeys. J Endod. 1997 Apr;23 (4):225-8.

12. Tziafas D, Pantelidou O, Alvanou A, Belibasakis G, Papadimitriou S. The dentinogenic effect of mineral trioxide aggregate (MTA) in short-term capping experiments. Int Endod J. 2002 Mar;35 (3):245-54.

13. Agamy HA, Bakry NS, Mounir MM, Avery DR. Comparison of mineral trioxide aggregate and formocresol as pulp-capping agents in pulpotomized primary teeth. Pediatr Dent. 2004 Jul-Aug;26(4): 302-9.

14. Farsi N, Alamoudi N, Balto K, Mushayt A. Success of mineral trioxide aggregate in pulpotomized primary molars. J Clin Pediatr Dent. 2005 Summer; 29(4):307-11.

15. Holan G, Eidelman E, Fuks AB. Long-term evaluation of pulpotomy in primary molars using 
mineral trioxide aggregate or formocresol.Pediatr Dent. 2005 Mar-Apr;27(2):129-36.

16. Naik S, Hegde AM. Mineral trioxide aggregate as a pulpotomy agent in primary molars: An in vivo study. J Indian Soc Pedod Prev Dent. 2005 Mar; 23(1):13-6.

17. Peng L, Ye L, Tan H, Zhou X. Evaluation of the formocresol versus mineral trioxide aggregate primary molar pulpotomy: a meta-analysis. Oral Surg Oral Med Oral Pathol Oral Radiol Endod. 2006 Dec;102(6):e40-4.

18. Aeinehchi M, Dadvand S, Fayazi S, BayatMovahed S. Randomized controlled trial of mi- neral trioxide aggregate and formocresol for pulpotomy in primary molar teeth. Int Endod $J$. 2007 Apr;40(4):261-7. Epub 2007 Feb 19.

19. Noorollahian H. Comparison of mineral trioxide aggregate and formocresol as pulp medicaments for pulpotomies in primary molars. $\mathrm{Br}$ Dent J. 2008 Jun 14;204(11):E20. Epub 2008 Apr 18.

\section{CORRESPONDENCIA}

Miguel Angel Simancas Pallares

Correo electrónico: simancaspallares@gmail.com. 